\title{
Fuzzy implications defined on the set of discrete fuzzy numbers
}

\author{
Juan Vicente Riera ${ }^{1}$ Joan Torrens ${ }^{1}$ \\ ${ }^{1}$ Department of Mathematics and Computer Science, University of the Balearic Islands. Spain.
}

\begin{abstract}
Given an implication function $I$ defined on the finite chain $L=\{0, \ldots, n\}$, a method for extending $I$ to the set of discrete fuzzy numbers whose support is a set of consecutive natural numbers contained in $L$ (denoted by $\mathcal{A}_{1}^{L}$ ) is given. The resulting extension is in fact a fuzzy implication on $A_{1}^{L}$ preserving some boundary properties. Moreover, if the initial implication $I$ is an $\mathrm{S}, \mathrm{QL}$ or D-implication on $L$ then its extension is also an $\mathrm{S}, \mathrm{QL}$ or D-implication on $\mathcal{A}_{1}^{L}$, respectively.
\end{abstract}

Keywords: Implication function, discrete fuzzy number, finite chain, lattice.

\section{Introduction}

Fuzzy implication functions play a fundamental role in fuzzy logic, fuzzy control and approximate reasoning. As a generalization of implications in classical logic, they are used not only to model fuzzy conditionals, but also in the inference process through the modus ponens and modus tollens rules ([1] or [12]). When we deal with fuzzy logic the used scale is always the unit interval $[0,1]$. However, in many applications only a finite number of values is used and even only qualitative information is handled. For this reason, many authors have studied in last years operations defined on a finite chain $L$, usually called discrete operations. For instance, t-norms and t-conorms were characterized in [16], uninorms and nullnorms in [13], weighted means in [11] and also implications functions in [14] and [15]. It is proved in [16] that only the number of elements of the finite chain $L$ is relevant when we deal with monotonic operations on $L$, and so the finite chain used in many of the mentioned works is the most simple one $L=\{0,1, \ldots, n\}$. As in the case of $[0,1]$, the four most usual ways to define fuzzy implications on the finite chain $L$ are the so-called $\mathrm{R}, \mathrm{S}$, QL, and D-implications (see [14] and [15]).

Recently, another approach deals with the possibility of extending monotonic operations on $L$ to operations on the set of discrete fuzzy numbers whose support is a set of consecutive natural numbers contained in $L$. More specifically, the concept of discrete fuzzy number was introduced in [17] as a fuzzy subset of $\mathbb{R}$ with discrete support and analogous properties to a fuzzy number. It is well known that arithmetic and lattice operations between fuzzy numbers are defined using the Zadeh's extension principle (see [10]). However, in general, for discrete fuzzy numbers this method fails and some approaches have been introduced in order to avoid such a drawback $([2,3,5]$ and $[18])$. In particular, it is proved in [5] that the set, $\mathcal{A}_{1}^{L}$, of discrete fuzzy numbers whose support is a set of consecutive natural numbers contained in $L$, is a distributive lattice. Thus, it is natural to study monotonic operations defined on $\mathcal{A}_{1}^{L}$ equipped with the usual lattice order. In this way, one approach is the one already commented of extending monotonic operations defined on $L$ to monotonic operations defined on the set $\mathcal{A}_{1}^{L}$. This was done for the special case of discrete t-norms and t-conorms in [6] and for the case of discrete means in [8].

Following with this idea we want to study in this paper the possibility of extending discrete implications on $L$ to implications defined on $\mathcal{A}_{1}^{L}$. Moreover, we will see that some boundary properties on fuzzy implications are preserved under this extension, as well as some types of implications. That is, the extension of an S-implication on $L$ leads to an Simplication on $\mathcal{A}_{1}^{L}$, and the same occurs for QL and $\mathrm{D}$-implications. The case of R-implications needs a deeper study which we want to develop as a future work.

\section{Preliminaries}

In this section we recall some results that will be used along the papers or we give references where they can be found.

\subsection{Discrete implications}

Let $(\mathcal{P}, \leq)$ be a bounded ordered set with smallest element 0 and greatest element 1.

Definition 2.1 [9] An implication function $I$ on $(\mathcal{P}, \leq)$ is a binary operator $I: \mathcal{P} \times \mathcal{P} \rightarrow \mathcal{P}$ that is decreasing in the first variable, increasing in the second one and satisfies the corner conditions $I(0,0)=1, I(1,1)=1$ and $I(1,0)=0$.

Remark 2.2 [9] Note that for any implication function I on $(\mathcal{P}, \leq)$ it turns out that

$$
I(0, \alpha)=I(\alpha, 1)=1, \text { for all } \alpha \in \mathcal{P}
$$

a property called the absorption principle. 
Definition 2.3 [9] A border implication I on $(\mathcal{P}, \leq$ ) is an implication function that satisfies the neutrality principle

$$
I(1, \beta)=\beta, \text { for all } \beta \in \mathcal{P} .
$$

Definition 2.4 Let I be an implication function on $(\mathcal{P}, \leq)$. Then I satisfies

i) the exchange principle $(E P)$, if

$$
I(x, I(y, z))=I(y, I(x, z)) \text { for all } x, y, z \in \mathcal{P}
$$

ii) the contraposition law $(C L)$ with respect to the strong negation $N$ on $\mathcal{P}$, if

$$
I(N(y), N(x))=I(x, y) \text { for all } x, y \in \mathcal{P}
$$

iii) the ordering property $(O P)$, if

$$
I(x, y)=1 \Leftrightarrow x \leq y, \text { for all } x, y \in \mathcal{P} .
$$

A particular case of bounded ordered set is when we consider the finite chain $L=\{0, \ldots, n\} \subset \mathbb{N}$. Operators defined on $L$ are usually called discrete operations and they have been studied by many authors (see $[9,11,13,16])$. In these studies the following condition, generally used as a discrete counterpart of continuity, is usually considered.

Definition 2.5 A function $f: L \rightarrow L$ is said to be smooth if it satisfies: $|f(x)-f(x-1)| \leq 1$ for all $x \in L$ with $x \geq 1$.

Definition 2.6 A binary operation $F: L^{2} \rightarrow L$ is said to be smooth when each one of its vertical and horizontal sections $(F(x,$.$) and F(., y)$, respectively) are smooth.

Smooth discrete t-norms and t-conorms were characterized in [16]. There, it is also proved that there is one and only one strong negation on $L$ which is given by

$$
N(x)=n-x \quad \text { for all } \quad x \in L .
$$

The four most usual ways to define implication functions on $L$ were investigated in [14] and [15]. That is,

$$
\begin{aligned}
& I(x, y)=\max \{z \in L \mid T(x, z) \leq y\} \text { (R-implications) } \\
& I(x, y)=S(N(x), y) \text { (S-implications) } \\
& I(x, y)=S(N(x), T(x, y)) \in L(\text { QL-implications) } \\
& I(x, y)=S(T(N(x), N(y)), y) \in L \text { (D-implications) }
\end{aligned}
$$

for all $x, y \in L$, where $T$ is a (smooth) t-norm, $S$ a (smooth) t-conorm and $N$ the strong negation on $L$ given by equation (1). From now on, $N$ will always denote such a negation.

\subsection{Discrete fuzzy numbers}

In this section, we recall some definitions and the main results about discrete fuzzy numbers which will be used later. By a fuzzy subset of $\mathbb{R}$, we mean a function $A: \mathbb{R} \rightarrow[0,1]$. For each fuzzy subset $A$, let $A^{\alpha}=\{x \in \mathbb{R}: A(x) \geq \alpha\}$ for any $\alpha \in(0,1]$ be its $\alpha$-level set (or $\alpha$-cut). By $\operatorname{supp}(A)$, we mean the support of $A$, i.e. the set $\{x \in \mathbb{R}: A(x)>0\}$. By $A^{0}$, we mean the closure of $\operatorname{supp}(A)$.

Definition 2.7 [17] A fuzzy subset $A$ of $\mathbb{R}$ with membership mapping $A: \mathbb{R} \rightarrow[0,1]$ is called discrete fuzzy number if its support is finite, i.e., there exist $x_{1}, \ldots, x_{n} \in \mathbb{R}$ with $x_{1}<x_{2}<\ldots<x_{n}$ such that $\operatorname{supp}(A)=\left\{x_{1}, \ldots, x_{n}\right\}$, and there are natural numbers $s, t$ with $1 \leq s \leq t \leq n$ such that:

1. $A\left(x_{i}\right)=1$ for any natural number $i$ with $s \leq i \leq$ $t$ (core)

2. $A\left(x_{i}\right) \leq A\left(x_{j}\right)$ for each natural number $i, j$ with $1 \leq i \leq j \leq s$

3. $A\left(x_{i}\right) \geq A\left(x_{j}\right)$ for each natural number $i, j$ with $t \leq i \leq j \leq n$

Remark 2.8 If the fuzzy subset $A$ is a discrete fuzzy number then the support of $A$ coincides with its closure, i.e. $\operatorname{supp}(A)=A^{0}$.

From now on, we will denote the set of discrete fuzzy numbers by $D F N$ and the abbreviation $d f n$ will denote a discrete fuzzy number.

Theorem 2.9 [18] (Representation of discrete fuzzy numbers) Let $A$ be a discrete fuzzy number. Then the following statements (1)-(4) hold:

1. $A^{\alpha}$ is a nonempty finite subset of $\mathbb{R}$, for any $\alpha \in[0,1]$

2. $A^{\alpha_{2}} \subseteq A^{\alpha_{1}}$ for any $\alpha_{1}, \alpha_{2} \in[0,1]$ with $0 \leq$ $\alpha_{1} \leq \alpha_{2} \leq 1$

3. For any $\alpha_{1}, \alpha_{2} \in[0,1]$ with $0 \leq \alpha_{1} \leq \alpha_{2} \leq 1$, if $x \in A^{\alpha_{1}}-A^{\alpha_{2}}$ we have $x<y$ for all $y \in A^{\alpha_{2}}$, or $x>y$ for all $y \in A^{\alpha_{2}}$

4. For any $\alpha_{0} \in(0,1]$, there exist some real numbers $\alpha_{0}^{\prime}$ with $0<\alpha_{0}^{\prime}<\alpha_{0}$ such that $A^{\alpha_{0}^{\prime}}=A^{\alpha_{0}}$ (i.e. $A^{\alpha}=A^{\alpha_{0}}$ for any $\alpha \in\left[\alpha_{0}^{\prime}, \alpha_{0}\right]$ ).

Theorem 2.10 [18] Conversely, if for any $\alpha \in$ $[0,1]$, there exists $A^{\alpha} \subset \mathbb{R}$ satisfying analogous conditions to the (1)-(4) of Theorem 2.9, then there exists a unique $A \in D F N$ such that its $\alpha$-cuts are exactly the sets $A^{\alpha}$ for any $\alpha \in[0,1]$.

\subsection{Maximum and minimum of discrete fuzzy numbers}

Let $A, B$ be two dfn and $A^{\alpha}=\left\{x_{1}^{\alpha}, \cdots, x_{p}^{\alpha}\right\}, B^{\alpha}=$ $\left\{y_{1}^{\alpha}, \cdots, y_{k}^{\alpha}\right\}$ their $\alpha$-cuts respectively. 
In [3], for each $\alpha \in[0,1]$, we consider the following sets,

$\min (A, B)^{\alpha}=\{z \in \operatorname{supp}(A) \wedge \operatorname{supp}(B)$ such that $\left.\min \left(x_{1}^{\alpha}, y_{1}^{\alpha}\right) \leq z \leq \min \left(x_{p}^{\alpha}, y_{k}^{\alpha}\right)\right\}$ and

$\max (A, B)^{\alpha}=\{z \in \operatorname{supp}(A) \vee \operatorname{supp}(B)$ such that $\left.\max \left(x_{1}^{\alpha}, y_{1}^{\alpha}\right) \leq z \leq \max \left(x_{p}^{\alpha}, y_{k}^{\alpha}\right)\right\}$

where $\operatorname{supp}(A) \wedge \operatorname{supp}(B)=$

$$
\{z=\min (x, y) \mid x \in \operatorname{supp}(A), y \in \operatorname{supp}(B)\}
$$

and $\operatorname{supp}(A) \vee \operatorname{supp}(B)=$

$$
\{z=\max (x, y) \mid x \in \operatorname{supp}(A), y \in \operatorname{supp}(B)\}
$$

Proposition 2.11 [3] There exist two unique discrete fuzzy numbers, that we will denote by $\operatorname{MIN}(A, B)$ and $M A X(A, B)$, such that they have the sets $\min (A, B)^{\alpha}$ and $\max (A, B)^{\alpha}$ as $\alpha$-cuts respectively.

The following result holds for $\mathcal{A}_{1}^{L}$, but is not true for the set of discrete fuzzy numbers in general(see [5]).

Theorem 2.12 [5] The triplet $\left(\mathcal{A}_{1}^{L}, M I N, M A X\right)$ is a bounded distributive lattice where $\mathbf{N} \in \mathcal{A}_{1}^{L}$ (the unique discrete fuzzy number whose support is the singleton $\{n\}$ ) and $\mathbf{O} \in \mathcal{A}_{1}^{L}$ (the unique discrete fuzzy number whose support is the singleton $\{0\}$ ) are the maximum and the minimum, respectively.

Remark 2.13 [5] Using these operations, we can define a partial order on $\mathcal{A}_{1}^{L}$ in the usual way: $A \preceq B$ if and only if $M I N(A, B)=A$, or equivalently, $A \preceq B$ if and only if $M A X(A, B)=B$ for any $A, B \in \mathcal{A}_{1}^{L}$. Equivalently, we can also define the partial ordering in terms of $\alpha$-cuts:

$A \preceq B$ if and only if $\min \left(A^{\alpha}, B^{\alpha}\right)=A^{\alpha}$

$A \preceq B$ if and only if $\max \left(A^{\alpha}, B^{\alpha}\right)=B^{\alpha}$

Smooth t-norms and t-conorms on $L$ were extended to t-norms and t-conorms on $\mathcal{A}_{1}^{L}$ in [6].

Theorem 2.14 [6] Let $T(S)$ be a smooth $t$-norm(tconorm) on $L$ and let

$$
\begin{aligned}
\mathcal{T}(\mathcal{S}): \mathcal{A}_{1}^{L} \times \mathcal{A}_{1}^{L} & \rightarrow \mathcal{A}_{1}^{L} \\
(A, B) & \longmapsto \mathcal{T}(\mathcal{S})(A, B)
\end{aligned}
$$

be the extension of t-norm(t-conorm) $T(S)$ to $\mathcal{A}_{1}^{L}$, defined as follows $\mathcal{T}(\mathcal{S})(A, B)$ is the discrete fuzzy number whose $\alpha$-cuts are the sets $\left\{z \in L \mid T(S)\left(\min A^{\alpha}, \min B^{\alpha}\right) \leq z \leq\right.$ $\left.T(S)\left(\max A^{\alpha}, \max B^{\alpha}\right)\right\}$ for each $\alpha \in[0,1]$. Then, $\mathcal{T}(\mathcal{S})$ is a t-norm (t-conorm) on the bounded set $\mathcal{A}_{1}^{L}$.

Similarly to Theorem 2.14 above, it is possible to extend the unique strong negation function $n$ defined on $L$ to a strong negation function $\mathcal{N}$ on the bounded lattice $\mathcal{A}_{1}^{L}$.
Proposition 2.15 [7] Let us consider the strong negation $N$ on the finite chain $L=\{0,1, \cdots, n\}$. The mapping

$$
\begin{aligned}
\mathcal{N}: \mathcal{A}_{1}^{L} & \longrightarrow \mathcal{A}_{1}^{L} \\
A & \mapsto \mathcal{N}(A)
\end{aligned}
$$

is a strong negation on the bounded distributive lattice $\mathcal{A}_{1}^{L}=\left(\mathcal{A}_{1}^{L}, M I N, M A X\right)$ where $\mathcal{N}(A)$ is the discrete fuzzy number such that has as support the sets $\mathcal{N}(A)^{\alpha}=\left[N\left(x_{p}^{\alpha}\right), N\left(x_{1}^{\alpha}\right)\right]$ for each $\alpha \in[0,1]$ (being $A^{\alpha}=\left[x_{1}^{\alpha}, x_{p}^{\alpha}\right]$ the $\alpha$-cuts of $A$ ).

\section{Implication functions on $\mathcal{A}_{1}^{L}$}

In this section we wish to study if it is possible to build implication functions on the bounded set $\mathcal{A}_{1}^{L}$ constructed from a discrete implication function $I$ defined on the finite chain $L$. Let us begin with some notation. If

$$
\begin{aligned}
O: L \times L & \longrightarrow L \\
(x, y) & \longmapsto O(x, y)
\end{aligned}
$$

is a binary discrete function on $L$ (e.g. a t-norm, t-conorm, implication, etc.), we will denote as well by $O$, the binary operation

$$
\begin{aligned}
O: 2^{L} \times 2^{L} & \longrightarrow 2^{L} \\
(\mathrm{X}, \mathrm{Y}) & \longmapsto O(\mathrm{X}, \mathrm{Y})
\end{aligned}
$$

where $O(\mathrm{X}, \mathrm{Y})=\{O(x, y) \mid x \in \mathrm{X}, y \in \mathrm{Y}\}$.

Lemma 3.1 Let us consider $A, B \in \mathcal{A}_{1}^{L}$. And let $I$ be a discrete implication function on the finite chain $L$. Then the following relations

$$
\begin{aligned}
\min I\left(A^{\alpha}, B^{\alpha}\right) & =I\left(\max A^{\alpha}, \min B^{\alpha}\right) \\
\max I\left(A^{\alpha}, B^{\alpha}\right) & =I\left(\min A^{\alpha}, \max B^{\alpha}\right)
\end{aligned}
$$

hold for each $\alpha \in[0,1]$, where $A^{\alpha}, B^{\alpha}$ are the $\alpha$-cut sets for $A$ and $B$ respectively.

Proof We only show the second relation because the proof of the first one is similar. Note that the inequality

$$
I\left(\min A^{\alpha}, \max B^{\alpha}\right) \leq \max I\left(A^{\alpha}, B^{\alpha}\right)
$$

is clear. To show the converse inequality, since $I$ is decreasing in the first variable and increasing in the second one, we have

$$
I(x, y) \leq I\left(\min A^{\alpha}, \max B^{\alpha}\right)
$$

for all $x \in A^{\alpha}$ and for all $y \in B^{\alpha}$. Thus

$$
\max I\left(A^{\alpha}, B^{\alpha}\right) \leq I\left(\min A^{\alpha}, \max B^{\alpha}\right) .
$$

Proposition 3.2 Let us consider $A, B \in \mathcal{A}_{1}^{L}$. And let $I$ be any discrete implication function on the finite chain $L$. There exists a unique discrete fuzzy number whose $\alpha$-cuts are exactly the sets $C^{\alpha}=$

$$
\left\{z \in L \mid \min I\left(A^{\alpha}, B^{\alpha}\right) \leq z \leq \max I\left(A^{\alpha}, B^{\alpha}\right)\right\}
$$

that will be denoted by $\mathcal{I}(A, B)$. Moreover, $\mathcal{I}(A, B) \in \mathcal{A}_{1}^{L}$. 
Proof Now, we will see that the sets $C^{\alpha}$ satisfy the four conditions of the Wang's Theorem (see Theorems 2.9 and 2.10 )

1. For each $\alpha \in[0,1], C^{\alpha}$ is a nonempty finite set, because of $A^{\alpha}$ and $B^{\alpha}$ are both nonempty finite sets (the discrete fuzzy numbers are normal fuzzy subsets).

2. $C^{\beta} \subseteq C^{\alpha}$ for any $\alpha, \beta \in[0,1]$ with $0 \leq \alpha \leq$ $\beta \leq 1$.

Because if $A, B \in \mathcal{A}_{1}^{L}$ we know that

$$
\begin{array}{rr}
A^{\beta} \subseteq A^{\alpha} \text { implies } & \min A^{\alpha} \leq \min A^{\beta} \\
\text { and } & \max A^{\beta} \leq \max A^{\alpha} \\
B^{\beta} \subseteq B^{\alpha} \text { implies } & \min B^{\alpha} \leq \min B^{\beta} \\
\text { and } & \max B^{\beta} \leq \max B^{\alpha}
\end{array}
$$

Moreover, as $I$ is decreasing in the first variable, using the relation (4) we obtain

$$
I\left(\max A^{\alpha}, z\right) \leq I\left(\max A^{\beta}, z\right) \text { for all } z \in L
$$

And, in particular by relation (5),

$$
I\left(\max A^{\alpha}, \min B^{\alpha}\right) \leq I\left(\max A^{\beta}, \min B^{\beta}\right)
$$

Thus, from Lemma 3.1

$$
\min I\left(A^{\alpha}, B^{\alpha}\right) \leq \min I\left(A^{\beta}, B^{\beta}\right)
$$

Similarly, we can see that

$$
\max I\left(A^{\beta}, B^{\beta}\right) \leq \min I\left(A^{\alpha}, B^{\alpha}\right) .
$$

Combining the previous conditions (7) and (8), we obtain $C^{\beta}=$

$=\left\{z \in L \mid \min I\left(A^{\beta}, B^{\beta}\right) \leq z \leq \max I\left(A^{\beta}, B^{\beta}\right)\right\}$ $\subseteq\left\{z \in L \mid \min I\left(A^{\alpha}, B^{\alpha}\right) \leq z \leq \max I\left(A^{\alpha}, B^{\alpha}\right)\right\}$ $=C^{\alpha}$

Therefore, $C^{\beta} \subseteq C^{\alpha}$.

3. If $x \in C^{\alpha}-C^{\beta}$ then $x \in L$ and $x$ does not belong to $C^{\beta}$, hence either $x<$ $I\left(\max A^{\beta}, \min B^{\beta}\right)$, which is the minimum of $C^{\beta}$, or $x>I\left(\min A^{\beta}, \max B^{\beta}\right)$, which is the maximum of $C^{\beta}$.

4. As $A, B \in \mathcal{A}_{1}^{L}$, according to Theorem 2.9, for each $\alpha \in(0,1]$ there exist real numbers $\alpha_{1}^{\prime}$ and $\alpha_{2}^{\prime}$ with $0<\alpha_{1}^{\prime}<\alpha$ and $0<\alpha_{2}^{\prime}<\alpha$ such that for each $r \in\left[\alpha_{1}^{\prime}, \alpha\right], A^{\alpha}=A^{r}$. Moreover $B^{\alpha}=$ $B^{r}$, for each $r \in\left[\alpha_{2}^{\prime}, \alpha\right]$. Thus, if $\alpha^{\prime}=\alpha_{1}^{\prime} \vee \alpha_{2}^{\prime}$, we can obtain:

$$
\begin{aligned}
& \min A^{r}=\min A^{\alpha} \text { and } \max A^{r}=\max A^{\alpha} \\
& \min B^{r}=\min B^{\alpha} \text { and } \max B^{r}=\max B^{\alpha}
\end{aligned}
$$

for each $r \in\left[\alpha^{\prime}, \alpha\right]$. Therefore

$$
\begin{aligned}
& I\left(\max A^{r}, \min B^{r}\right)=I\left(\max A^{\alpha}, \min B^{\alpha}\right) \\
& I\left(\min A^{r}, \max B^{r}\right)=I\left(\min A^{\alpha}, \max B^{\alpha}\right) .
\end{aligned}
$$

Hence, by Lemma 3.1

$$
\begin{aligned}
\min I\left(A^{r}, B^{r}\right) & =\min I\left(A^{\alpha}, B^{\alpha}\right) \\
\max I\left(A^{r}, B^{r}\right) & =\max I\left(A^{\alpha}, B^{\alpha}\right)
\end{aligned}
$$

$$
\begin{aligned}
& \text { and so, } C^{r}= \\
& =\left\{z \in L \mid \min I\left(A^{r}, B^{r}\right) \leq z \leq \max I\left(A^{r}, B^{r}\right)\right\} \\
& =\left\{z \in L \mid \min I\left(A^{\alpha}, B^{\alpha}\right) \leq z \leq \max I\left(A^{\alpha}, B^{\alpha}\right)\right\} \\
& =C^{\alpha} \text { for each } r \in\left[\alpha^{\prime}, \alpha\right] .
\end{aligned}
$$

As the sets $C^{\alpha}$ fulfill for each $\alpha \in[0,1]$ the conditions stated in Theorem 2.9, by Theorem 2.10 there exists a unique discrete number, that will be denoted by $\mathcal{I}(A, B)$, such that its $\alpha$-cuts are exactly these sets.

In addition, from the construction of the sets $C^{\alpha}$ for each $\alpha \in[0,1]$, it is straightforward to see that are sets of consecutive natural numbers belonging to the finite chain $L$. Thus, $\mathcal{I}(A, B) \in \mathcal{A}_{1}^{L}$.

The previous proposition will allow us to define a binary operation $\mathcal{I}$ on $\mathcal{A}_{1}^{L}$ from an implication function $I$ defined on the finite chain $L$.

Definition 3.3 Let us consider an implication function $I$ on the finite chain $L$. The binary operation on $\mathcal{A}_{1}^{L}$ defined as follows

$$
\begin{aligned}
\mathcal{I}: \mathcal{A}_{1}^{L} \times \mathcal{A}_{1}^{L} & \longrightarrow \mathcal{A}_{1}^{L} \\
(A, B) & \longmapsto \mathcal{I}(A, B)
\end{aligned}
$$

will be called the extension of the discrete implication function $I$ to $\mathcal{A}_{1}^{L}$, being $\mathcal{I}(A, B)$ the discrete fuzzy number whose $\alpha$-cuts are the sets

$$
\left\{z \in L \mid \min I\left(A^{\alpha}, B^{\alpha}\right) \leq z \leq \max I\left(A^{\alpha}, B^{\alpha}\right)\right\}
$$

for each $\alpha \in[0,1]$.

Now we wish to study if the extension of the discrete implication function $I$ to $\mathcal{A}_{1}^{L}$ defined above satisfies the condition to be a implication function on the bounded set $\mathcal{A}_{1}^{L}$ according to Definition 2.1.

Theorem 3.4 Let I be an implication function on $L$. Then the extension of the discrete implication function $I, \mathcal{I}$ is an implication function on $\mathcal{A}_{1}^{L}$.

Proof To show this result, we will prove all the conditions stated in Definition 2.1. Let us consider $A, B \in \mathcal{A}_{1}^{L}$.

- We wish to see that $\mathcal{I}$ is decreasing in the first variable, i.e. if $A \preceq B$ then $\mathcal{I}(A, C) \succeq$ $\mathcal{I}(B, C)$ for all $C \in \mathcal{A}_{1}^{L}$. According to Remark 2.13 is equivalent to prove that $\min \left(\mathcal{I}(B, C)^{\alpha}, \mathcal{I}(A, C)^{\alpha}\right)=\mathcal{I}(B, C)^{\alpha}$ for all $\alpha \in[0,1]$. As $A \preceq B$ then $\min A^{\alpha} \leq \min B^{\alpha}$ and $\max A^{\alpha} \leq \max B^{\alpha}$. Thus as $I$ is an implication function on $L$, it is a decreasing function in the first variable, then by Lemma 3.1

$$
\begin{aligned}
\min I\left(A^{\alpha}, C^{\alpha}\right) & =I\left(\max A^{\alpha}, \min C^{\alpha}\right) \\
& \geq I\left(\max B^{\alpha}, \min C^{\alpha}\right) \\
& =\min I\left(B^{\alpha}, C^{\alpha}\right)
\end{aligned}
$$


Analogously,

$$
\begin{aligned}
\max I\left(A^{\alpha}, C^{\alpha}\right) & =I\left(\min A^{\alpha}, \max C^{\alpha}\right) \\
& \geq I\left(\min B^{\alpha}, \max C^{\alpha}\right) \\
& =\max I\left(B^{\alpha}, C^{\alpha}\right)
\end{aligned}
$$

Finally using the relations (9) and (10) above $\mathcal{I}(A, C)^{\alpha}=\left\{z \in L \mid \min I\left(A^{\alpha}, C^{\alpha}\right) \leq\right.$ $\left.z \leq \max I\left(A^{\alpha}, C^{\alpha}\right)\right\} \geq\{z \in L$ $\left.\min I\left(B^{\alpha}, C^{\alpha}\right) \leq z \leq \max I\left(B^{\alpha}, C^{\alpha}\right)\right\}=$ $\mathcal{I}(B, C)^{\alpha}$ for each $\alpha \in[0,1]$.

- The increasingness with the second variable follows similarly.

- With respect to the boundary conditions we have $\mathcal{I}(\mathbf{O}, \mathbf{O})^{\alpha}=\left\{z \in L \mid \min I\left(\mathbf{O}^{\alpha}, \mathbf{O}^{\alpha}\right) \leq\right.$ $\left.z \leq \max I\left(\mathbf{O}^{\alpha}, \mathbf{O}^{\alpha}\right)\right\}=\{n\}=\mathbf{N}^{\alpha}$ because of $\min I\left(\mathbf{O}^{\alpha}, \mathbf{O}^{\alpha}\right)=\max I\left(\mathbf{O}^{\alpha}, \mathbf{O}^{\alpha}\right)=n$ for all $\alpha \in[0,1]$. Then $\mathcal{I}(\mathbf{O}, \mathbf{O})=\mathbf{N}$.

The other two conditions $\mathcal{I}(\mathbf{N}, \mathbf{N})=\mathbf{N}$ and $\mathcal{I}(\mathbf{N}, \mathbf{O})=\mathbf{0}$ follows similarly.

Remark 3.5 Note that, since $\mathcal{I}$ is an implication on $\mathcal{A}_{1}^{L}$, it must also satisfy the absorption property, that is,

$$
\mathcal{I}(\mathbf{O}, A)=\mathcal{I}(A, \mathbf{N})=\mathbf{N}
$$

for all $a \in \mathcal{A}_{1}^{L}$.

Example 3.6 Let us consider $L=$ $\{0,1,2,3,4,5,6\}$ and

$$
\begin{aligned}
& A=\{0.3 / 0,0.5 / 1,1 / 2,0.8 / 3,0.5 / 4\} \\
& B=\{0.6 / 2,0.8 / 3,0.9 / 4,1 / 5,0.8 / 6\}
\end{aligned}
$$

belonging to $\mathcal{A}_{1}^{L}$. If we consider the implication functions

$I_{R}(x, y)=\left\{\begin{array}{l}6 \text { if } x \leq y \\ 3+y-x \text { if there exist } 0 \leq y<x \leq 3 \\ 6+y-x \text { if there exist } 3 \leq y<x \leq 6 \\ y \text { otherwise }\end{array}\right.$

$I_{S}(x, y)=\left\{\begin{array}{l}\min (6,3+y-x) \text { if } x \in[0,3], y \in[3,6] \\ \min (3,6+y-x) \text { if } x \in[3,6], y \in[0,3] \\ \max (6-x, y) \text { otherwise }\end{array}\right.$

we obtain that

$$
\begin{aligned}
& \mathcal{I}_{R}(A, B)=\{0.6 / 2,0.6 / 3,0.6 / 4,0.6 / 5,1 / 6\} \\
& \mathcal{I}_{S}(A, B)=\{0.8 / 3,0.8 / 4,0.9 / 5,1 / 6\}
\end{aligned}
$$

In the next two propositions we deal with some other properties that are many times required for implications, depending on the context.

Proposition 3.7 If an implication function $I$ is a border implication on $L$ then implication $\mathcal{I}$ generated by $I$ according to Definition 3.3 is a border implication on the bounded set $\mathcal{A}_{1}^{L}$ as well.
Proof To show this condition it is enough to see that $\mathcal{I}(\mathbf{N}, B)^{\alpha}=$

$$
\begin{aligned}
& \left\{z \in L \mid \min I\left(\mathbf{N}^{\alpha}, B^{\alpha}\right) \leq z \leq \max I\left(\mathbf{N}^{\alpha}, B^{\alpha}\right)\right\} \\
= & \left\{z \in L \mid I\left(n, \min B^{\alpha}\right) \leq z \leq I\left(n, \max B^{\alpha}\right)\right\} \\
= & \left\{z \in L \mid \min B^{\alpha} \leq z \leq \max B^{\alpha}\right\} \\
= & B^{\alpha} \text { for each } \alpha \in[0,1] . \square
\end{aligned}
$$

Proposition 3.8 Let $I$ be an implication function on $L$ and $\mathcal{I}$ its extension on $\mathcal{A}_{1}^{L}$.

i) I satisfies $(E P)$ if and only if $\mathcal{I}$ satisfies $(E P)$.

ii) I satisfies $(C L)$ if and only if $\mathcal{I}$ satisfies $(C L)$.

iii) If I satisfies $(O P)$ then $n \in \mathcal{I}(A, B)^{\alpha}$ for all $\alpha \in[0,1]$, being $A, B \in \mathcal{A}_{1}^{L}$ with $A \preceq B$. Moreover, when $\max \operatorname{supp}(A) \leq \min \operatorname{supp}(B)$ then $\mathcal{I}(A, B)=\mathbf{N}$.

Proof Now, we will study each case.

i) Suppose that $I$ satisfies $(E P)$ and consider $A, B, C \in \mathcal{A}_{1}^{L}$. It is enough to show that

$$
\mathcal{I}(A, \mathcal{I}(B, C))^{\alpha}=\mathcal{I}(B, \mathcal{I}(A, C))^{\alpha},
$$

or equivalently, that

$$
\min I\left(A^{\alpha}, \mathcal{I}(B, C)^{\alpha}\right)=\min I\left(B^{\alpha}, \mathcal{I}(A, C)^{\alpha}\right)
$$

and

$$
\max I\left(A^{\alpha}, \mathcal{I}(B, C)^{\alpha}\right)=\max I\left(B^{\alpha}, \mathcal{I}(A, C)^{\alpha}\right) .
$$

However, applying Lemma 3.1 the minimums in the condition above are given by $\quad I\left(\max A^{\alpha}, I\left(\max B^{\alpha}, \min C^{\alpha}\right)\right) \quad$ and $I\left(\max B^{\alpha}, I\left(\max A^{\alpha}, \min C^{\alpha}\right)\right), \quad$ respectively, which coincide because $I$ satisfies $(E P)$.

Conversely, suppose that $\mathcal{I}$ satisfies $(E P)$ and consider $a, b, c \in L$. Let $1_{a}, 1_{b}$ and $1_{c}$ the discrete fuzzy numbers whose support is given by the singletons $\{a\},\{b\},\{c\}$, respectively. Then clearly

$$
\mathcal{I}\left(1_{a}, \mathcal{I}\left(1_{b}, 1_{c}\right)\right)=\mathcal{I}\left(1_{b}, \mathcal{I}\left(1_{a}, 1_{c}\right)\right)
$$

and this implies directly that $I$ satisfies $(E P)$.

ii) Similarly as above, for all $\alpha \in[0,1]$ and $A, B \in$ $\mathcal{A}_{1}^{L}$ we have

$$
\begin{aligned}
\min I\left(\mathcal{N}(B)^{\alpha}, \mathcal{N}(A)^{\alpha}\right) & =\min I\left(A^{\alpha}, B^{\alpha}\right) \\
\max I\left(\mathcal{N}(B)^{\alpha}, \mathcal{N}(A)^{\alpha}\right) & =\max I\left(A^{\alpha}, B^{\alpha}\right)
\end{aligned}
$$

and then

$$
\mathcal{I}(\mathcal{N}(B), \mathcal{N}(A))^{\alpha}=\mathcal{I}(A, B)^{\alpha}
$$

for all $\alpha \in[0,1]$.

The converse is again similar to the previous step. 
iii) If $A \preceq B$ then $\min A^{\alpha} \leq \max A^{\alpha} \leq \max B^{\alpha}$ for all $\alpha \in[0,1]$ and so $I\left(\min A^{\alpha}, \max B^{\alpha}\right)=n$. Therefore $n \in \mathcal{I}(A, B)^{\alpha}$ for all $\alpha \in[0,1]$.

Moreover, when $\max A^{\alpha} \leq \min B^{\alpha}$ we also have $I\left(\max A^{\alpha}, \min B^{\alpha}\right)=n$ and so, $\mathcal{I}(A, B)^{\alpha}=\{z \in L \mid n \leq z \leq n\}=\{n\}$ for all $\alpha \in[0,1]$, i.e., $\mathcal{I}(A, B)=\mathbf{N}$.

Example 3.9 Let us consider the Łukasiewicz implication on $L=\{0,1,2,3,4,5,6\}$ given by the expression $I(x, y)=\min (6,6+y-x)$. It is known that $I$ fulfills $(E P),(C L)$ and $(O P)$. Let us consider its extension $\mathcal{I}$ to $\mathcal{A}_{1}^{L}$ and the discrete fuzzy numbers

$$
\begin{aligned}
A & =\{0.6 / 0,0.7 / 1,1 / 2,0.8 / 3\} \\
B & =\{0.8 / 3,1 / 4,0.7 / 5,0.6 / 6\} \\
C & =\{0.6 / 0,0.7 / 1,0.8 / 2,1 / 3,0.8 / 4\} \\
D & =\{0.7 / 1,0.8 / 2,1 / 3,1 / 4,0.8 / 5\} .
\end{aligned}
$$

i) By the previous proposition $\mathcal{I}$ verifies $(C L)$. Thus, it can be trivially verified that

$$
\begin{aligned}
\mathcal{I}(\mathcal{N}(D), \mathcal{N}(C)) & =\mathcal{I}(C, D) \\
& =\{0.7 / 3,0.8 / 4,0.8 / 5,1 / 6\}
\end{aligned}
$$

ii) It is easy to see that $C \preceq D$. Thus, according to Proposition 3.8-iii) above,

$$
6 \in \operatorname{supp} \mathcal{I}(C, D)^{\alpha} \text { for all } \alpha \in[0,1],
$$

but note that $\mathcal{I}(C, D) \neq \mathbf{N}$. On the other hand, it is immediate to see that $A \preceq B$ and $\max \operatorname{supp}(A) \leq \min \operatorname{supp}(B)$. Thus, in this case we have $\mathcal{I}(A, B)=\{1 / 6\}=\mathbf{N}$.

Fuzzy negations can be build from fuzzy implication [1]. Similarly it is possible to obtain a negation on the partially ordered set $\mathcal{A}_{1}^{L}$.

Proposition 3.10 Let $\mathcal{I}$ an implication on $\mathcal{A}_{1}^{L}$. Then the function

$$
\begin{aligned}
\mathcal{N}_{\mathcal{I}}: & \mathcal{A}_{1}^{L} \\
A & \longmapsto \mathcal{A}_{1}^{L} \\
A & \mapsto \mathcal{N}_{\mathcal{I}}(A)=\mathcal{I}(A, \mathbf{O}),
\end{aligned}
$$

is a fuzzy negation on $\mathcal{A}_{1}^{L}$.

\section{Proof Straightforward.}

It is well known that the comparison of implication functions defined on a finite chain $L$ is done in the usual way, i.e., pointwise. In the same way, if we consider two implications functions $\mathcal{I}_{1}, \mathcal{I}_{2}$ on $\mathcal{A}_{1}^{L}$ generated by two implications $I_{1}, I_{2}$ on $L$ with $I_{1} \leq I_{2}$ it is possible to obtain a similar order between $\mathcal{I}_{1}$ and $\mathcal{I}_{2}$. So,

Proposition 3.11 Let $I_{1}$ and $I_{2}$ be two implications functions on the finite chain $L=\{0,1, \cdots, n\}$ verifying $I_{1}(x, y) \leq I_{2}(x, y)$ for all $(x, y) \in L \times L$. Let us consider their extensions on $\mathcal{A}_{1}^{L}, \mathcal{I}_{1}$ and $\mathcal{I}_{2}$ for $I_{1}$ and $I_{2}$ respectively. Then $\mathcal{I}_{1}(A, B) \preceq$ $\mathcal{I}_{2}(A, B)$ for all $A, B \in \mathcal{A}_{1}^{L}$.
Proof It is enough to prove that (see Remark 2.13) $\min \left(\mathcal{I}_{1}(A, B)^{\alpha}, \mathcal{I}_{2}(A, B)^{\alpha}\right)=\mathcal{I}_{1}(A, B)^{\alpha}$ for each $\alpha \in[0,1]$. To do it, note that

$$
\begin{aligned}
\min \mathcal{I}_{1}(A, B)^{\alpha} & =\min I_{1}\left(A^{\alpha}, B^{\alpha}\right) \\
& =I_{1}\left(\max A^{\alpha}, \min B^{\alpha}\right) \\
& \leq I_{2}\left(\max A^{\alpha}, \min B^{\alpha}\right) \\
& =\min I_{2}\left(A^{\alpha}, B^{\alpha}\right),
\end{aligned}
$$

and similarly for the maximums. This already implies

$$
\min \left(\mathcal{I}_{1}(A, B)^{\alpha}, \mathcal{I}_{2}(A, B)^{\alpha}\right)=\mathcal{I}_{1}(A, B)^{\alpha} .
$$

Recently, a method to build S-implications on the bounded set $\mathcal{A}_{1}^{L}$ has been proposed in [7]. The method consists in the usual way of defining the S-implication from a t-conorm $\mathcal{S}$ (the extension of a smooth t-conorm $S$ defined on the finite chain $L$ ) and the strong negation $\mathcal{N}$ (the extension of the strong negation $N$ on $L$ ). Now, we want to see that any of these S-implications are, in fact, the extension of an S-implication on $L$ using the general method to build an implication function on $\mathcal{A}_{1}^{L}$, presented in this paper (see Definition 3.3). Specifically,

Proposition 3.12 ([7]) Let $S$ be a smooth $t$ conorm on $L$ and let $\mathcal{S}$ and $\mathcal{N}$ be the extensions of $S$ and $N$ to $\mathcal{A}_{1}^{L}$, respectively. Let $\mathcal{I}_{\mathcal{S}, \mathcal{N}}$ be the mapping given by

$$
\begin{aligned}
\mathcal{I}_{\mathcal{S}, \mathcal{N}}: \mathcal{A}_{1}^{L} \times \mathcal{A}_{1}^{L} & \longrightarrow \mathcal{A}_{1}^{L} \\
(A, B) & \mapsto \mathcal{I}_{\mathcal{S}, \mathcal{N}}=\mathcal{S}(\mathcal{N}(A), B),
\end{aligned}
$$

where $\mathcal{S}(\mathcal{N}(A), B)$ is the discrete fuzzy number whose $\alpha$-cuts are the sets of consecutive natural numbers

$\left[S\left(N\left(\max A^{\alpha}\right), \min B^{\alpha}\right), S\left(N\left(\min A^{\alpha}\right), \max B^{\alpha}\right)\right]$

for each $\alpha \in[0,1]$. Then $\mathcal{I}_{\mathcal{S}, \mathcal{N}}$ is an implication function on $\mathcal{A}_{1}^{L}$, that is called the $S$-implication generated from $\mathcal{S}$ and $\mathcal{N}$.

Proposition 3.13 Let $S$ be a smooth t-conorm on $L$ and $I$ the discrete $S$-implication on $L$ defined from $S$ and $N$. Let $\mathcal{S}, \mathcal{N}$ and $\mathcal{I}$ be the extensions of $S$, $N$ and $I$ to $\mathcal{A}_{1}^{L}$, respectively. Then $\mathcal{I}=\mathcal{I}_{\mathcal{S}, \mathcal{N}}$.

Proof Let us consider $A, B \in \mathcal{A}_{1}^{L}$ and $A^{\alpha}, B^{\alpha}$ their $\alpha$-cuts for each $\alpha \in[0,1]$ respectively. Thus, by Definition 3.3 we have $\mathcal{I}(A, B)^{\alpha}=\{z \in L$ $\left.I\left(\max A^{\alpha}, \min B^{\alpha}\right) \leq z \leq I\left(\min A^{\alpha}, \max B^{\alpha}\right)\right\}$. As $I(a, b)=S(N(a), b)$ for all $a, b \in L$ we obtain $\mathcal{I}(A, B)^{\alpha}=\left\{z \in L \mid S\left(N\left(\max A^{\alpha}\right), \min B^{\alpha}\right) \leq\right.$ $\left.z \leq S\left(N\left(\min A^{\alpha}\right), \max B^{\alpha}\right)\right\}=\mathcal{S}(\mathcal{N}(A), B)^{\alpha}=$ $\mathcal{I}_{\mathcal{S}, \mathcal{N}}(A, B)^{\alpha}$ for each $\alpha \in[0,1]$.

\subsection{QL and D-implications on $\mathcal{A}_{1}^{L}$}

We want to see in this section that the behavior of QL and D-implications is similar to the case of Simplications. Recall that QL and D-operators need not to be implications. So, let us deal with QL and D-operators in general. 
Proposition 3.14 Let us consider a smooth $t$ norm $T$ and a smooth t-conorm $S$ on $L$, and $I$ the $Q L$-operator obtained from them by the expression $I(x, y)=S(N(x), T(x, y))$, for all $x, y \in L . W e$ can build the $Q L$-operator $\mathcal{I}$ on $\mathcal{A}_{1}^{L}$ given by

$$
\mathcal{I}(A, B)=\mathcal{S}(\mathcal{N}(A), \mathcal{T}(A, B)), \text { for all } A, B \in \mathcal{A}_{1}^{L} \text {. }
$$

Then $\mathcal{I}$ is an implication function on $\mathcal{A}_{1}^{L}$ if and only if $I$ is an implication on $L$.

Proof It is well known that any QL-operator on $L$ satisfies all properties of a fuzzy implication except perhaps the decreasingness with the first variable (see [15]), and the same proof holds for QLoperators on $\mathcal{A}_{1}^{L}$. Thus, we only need to prove that $I$ is decreasing in the first place if and only if so is $\mathcal{I}$.

On the one hand, if $I$ is decreasing with the first variable then $I$ is an implication and we can obtain its extension to $\mathcal{A}_{1}^{L}$. Now, a similar proof as in Proposition 3.13 shows that such extension coincides with the QL-operator $\mathcal{I}$, that is, $\mathcal{I}$ is an implication and consequently it is decreasing with the first variable.

Reciprocally, let us suppose that $\mathcal{I}$ is decreasing with the first variable and take $a, b, c \in L$ such that $a \leq b$. Consider $A, B, C \in \mathcal{A}_{1}^{L}$ the discrete fuzzy numbers whose support are the singletons $\{a\},\{b\}$ and $\{c\}$, respectively. It is clear that $A \preceq B$ and by decreasingness of $\mathcal{I}$ we have

$$
\mathcal{I}(B, C) \preceq \mathcal{I}(A, C)
$$

or equivalently

$$
\operatorname{MIN}(\mathcal{I}(A, C), \mathcal{I}(B, C))=\mathcal{I}(B, C) .
$$

Note that for all $\alpha \in[0,1]$ the $\alpha$-cuts of $\mathcal{I}(A, C)$ and $\mathcal{I}(B, C)$ are $I(a, c)$ and $I(b, c)$, respectively. Consequently, equation (11) leads to

$$
\min (I(a, c), I(b, c))=I(b, c),
$$

that is, $I(b, c) \leq I(a, c)$, proving that $I$ is decreasing with the first variable.

We have also a similar result for D-operators.

Proposition 3.15 Let us consider a smooth $t$ norm $T$ and a smooth $t$-conorm $S$ on $L$, and $I$ the $D$-operator obtained from them by the expression $I(x, y)=S(y, T(N(x), N(y)))$, for all $x, y \in L$. We can build the $Q L$-operator $\mathcal{I}$ on $\mathcal{A}_{1}^{L}$ given by

$\mathcal{I}(A, B)=\mathcal{S}(B, \mathcal{T}(\mathcal{N}(A), \mathcal{N}(B)))$, for all $A, B \in \mathcal{A}_{1}^{L}$.

Then $\mathcal{I}$ is an implication function on $\mathcal{A}_{1}^{L}$ if and only if $I$ is an implication on $L$.

Proof In this case, the only property that can fail of the definition of implication is the increasigness with respect to the second variable. Now, similarly as in the proposition above, it can be proved that $I$ is increasing in the second variable if and only if so is $\mathcal{I}$, showing the proposition.

QL and D-implications defined on $L$ were studied and characterized in [15]. In the case of QLimplications the following characterization was obtained:

Proposition 3.16 [15] Let us consider a smooth t-norm $T$, a smooth t-conorm $S$ and $I$ the $Q L$ operator obtained from them by the expression $I(x, y)=S(N(x), T(x, y))$, for all $x, y \in L$. Then, the following statements are equivalent:

(i) $I: L^{2} \rightarrow L$ is a QL-implication.

(ii) $S(N(x), x)=n$ for all $x \in L$.

(iii) $S$ is the Archimedean t-conorm given by $S(x, y)=\min (n, x+y)$ for all $x, y \in L$.

Moreover, in this case, $I$ is simply given by $I(x, y)=$ $N(x)+T(x, y)$ for all $x, y \in L$.

Similarly, it is possible to obtain a characterization of D-implications,

Proposition 3.17 [15] Let us consider a smooth t-norm $T, a$ smooth t-conorm $S$ and $I$ the $D$-operator given by the expression $I(x, y)=$ $S(T(N(x), N(y)), y)$, for all $x, y \in L$. Then, the following statements are equivalent:

(i) $I: L^{2} \rightarrow L$ is a D-implication.

(ii) $S$ is the Archimedean t-conorm given by $S(x, y)=\min (n, x+y)$ for all $x, y \in L$.

Moreover, in this case, $I$ is simply given by $I(x, y)=$ $y+T(N(x), N(y))$ for all $x, y \in L$.

Thus, using the previous propositions it is clear that we can build a QL-implication and a Dimplication on the bounded lattice $\mathcal{A}_{1}^{L}$ from the corresponding QL and D-implication defined on $L$, satisfying the conditions of Proposition 3.16 and Proposition 3.17, respectively.

Proposition 3.18 Let $T$ be a smooth t-norm on $L$, $\mathcal{T}$ the extension of $T$ and $\mathcal{N}$ the extension of $N$ to $\mathcal{A}_{1}^{L}$. Then the binary operation

$$
\begin{aligned}
\mathcal{I}_{q l}: & \mathcal{A}_{1}^{L} \times \mathcal{A}_{1}^{L} \longrightarrow \mathcal{A}_{1}^{L} \\
& (A, B) \longmapsto \mathcal{I}_{q l}(A, B)=\mathcal{N}(A) \oplus \mathcal{T}(A, B),
\end{aligned}
$$

(where $\oplus$ denotes the addition of discrete fuzzy numbers according to the Zadeh's extension principle [4, 10]) is the only QL-implication function on $\mathcal{A}_{1}^{L}$ which is an extension of a $Q L$-implication on $L$.

Proof It is clear from Propositions 3.14 and 3.16 that the only QL-implication function on $\mathcal{A}_{1}^{L}$ that is an extension of a QL-implication on $L$ is the extension of the implication

$$
I(x, y)=N(x)+T(x, y) .
$$


Now, a simple calculation similar to the one given in Proposition 3.13 shows that such extension is given by $\mathcal{I}_{q l}$.

Analogously,

Proposition 3.19 Let $T$ be a smooth $t$-norm on $L$, $\mathcal{T}$ the extension of $T$ and $\mathcal{N}$ the extension of $N$ to $\mathcal{A}_{1}^{L}$. Then the binary operation

$$
\begin{aligned}
\mathcal{I}_{n q l} & : \mathcal{A}_{1}^{L} \times \mathcal{A}_{1}^{L} \longrightarrow \mathcal{A}_{1}^{L} \\
& (A, B) \longmapsto \mathcal{I}_{n q l}(A, B)=B \oplus \mathcal{T}(\mathcal{N}(A), \mathcal{N}(B))
\end{aligned}
$$

is the only D-implication function on $\mathcal{A}_{1}^{L}$ which is an extension of a D-implication on $L$.

Proof Similar to the proof of the previous proposition.

\section{Conclusion}

In this article we have proposed a method to extend an implication function $I$ defined in a finite chain $L$ to the set $\mathcal{A}_{1}^{L}$ (of discrete fuzzy numbers whose support is a subset of consecutive natural numbers belonging to $L$ ). Moreover, we have shown that this extension $\mathcal{I}$ is an implication function that fulfills similar properties (boundary properties, absorption property, contraposition property, etc.) to the initial implication $I$. Finally, we have seen that the extension of the three most usual implications on $L$, that is, $S$-implication, $Q L$-implications and $D$-implication lead to an $S$-implication, $Q L$ implications and $D$-implication on $\mathcal{A}_{1}^{L}$. As a future work, we will study the case of $R$-implications and their properties.

\section{Acknowledgment}

This work has been partially supported by the MTM2009-10962 and MTM2009-10320 project grants, both with FEDER support.

\section{References}

[1] M. Baczyński and B. Jayaram, Fuzzy Implications, Studies in Fuzziness and Soft Computing, vol. 231, Springer, Berlin Heidelberg, 2008.

[2] J. Casasnovas and J.V. Riera, On the addition of discrete fuzzy numbers. WSEAS Transactions on Mathematics, 5(5): 549-554, WSEAS Press, 2006.

[3] J. Casasnovas and J.V. Riera, Maximum and Mininum of Discrete Fuzzy numbers. In Frontiers in Artificial Intelligence and Applications: artificial intelligence research and development, 163, pages 273-280, IOS Press, 2007.

[4] J. Casasnovas and J.V. Riera, Discrete fuzzy numbers defined on a subset of natural numbers, Theoretical Advances and Applications of Fuzzy Logic and Soft Computing, in the series: Advances in Soft Computing 42, pp. 573-582, Springer, 2007.
[5] J. Casasnovas and J.V. Riera, Lattice properties of discrete fuzzy numbers under extended min and max. In Proceedings IFSA-EUSFLAT, pages 647-652, July, Lisbon (Portugal), 2009.

[6] J. Casasnovas and J.V. Riera, Extension of discrete t-norms and t-conorms to discrete fuzzy numbers, Fuzzy Sets and Systems, 167(1):65-81, Elsevier, 2011.

[7] J. Casasnovas and J.V. Riera, S-implications in the set of discrete fuzzy numbers. In Proceedings IEEE-WCCI 2010, pages 2741-2747, July, Barcelona (Spain), 2010.

[8] J. Casasnovas and J.V. Riera, Weighted means of subjective evaluations. In R. Seising, V. Sanz (editors), Soft Computing in Humanities and Social Sciences. Springer-Verlag, Berlin. Forthcoming.

[9] B. De Baets, Model implicators and their characterization. In N. Sreele, editor, proceedings of the First ICSC International Symposium on Fuzzy logic, (ICSC 1995), pages A42-A49, Academic Press, 1995.

[10] G.J. Klir and B. Yuan, Fuzzy sets and fuzzy logic (Theory and applications). Prentice Hall, 1995.

[11] A. Kolesarova, G. Mayor and R. Mesiar, Weighted ordinal means, Information Sciences, 177: 3822-3830, Elsevier, 2007.

[12] M. Mas, M. Monserrat, J. Torrens and E. Trillas, A survey on Fuzzy Implications Functions, IEEE Transactions on fuzzy systems, 15: 11071121, IEEE Computational Intelligence Society, 2007.

[13] M. Mas, G. Mayor and J. Torrens, t-operators and uninorms on a finite totally ordered set, International Journal of Intelligent Systems, 14: 909-922, Wiley Periodicals, 1999.

[14] M. Mas, M. Monserrat and J. Torrens, Simplications and R-implications on a finite chain, Kybernetica, 40: 3-20, ÚTIA, 2004.

[15] M. Mas, M. Monserrat, J. Torrens, On two types of discretes implications, International Journal of Approximate Resoning, 40: 262-279, Elsevier, 2005.

[16] G. Mayor and J. Torrens, Triangular norms on discrete settings. In E.P. Klement and R. Mesiar, editors, Logical, Algebraic, Analytic, and Probabilistic Aspects of Triangular Norms, Elsevier, Netherlands, 2005, pages 189-230.

[17] W. Voxman, Canonical representations of discrete fuzzy numbers, Fuzzy Sets and Systems, 54: 457-466, Elsevier, 2001.

[18] G. Wang, C. Wu and C. Zhao, Representation and Operations of discrete fuzzy numbers. Southeast Asian Bulletin of Mathematics 28: 1003-1010, Elsevier, 2005. 